Pacific Journal of Mathematics

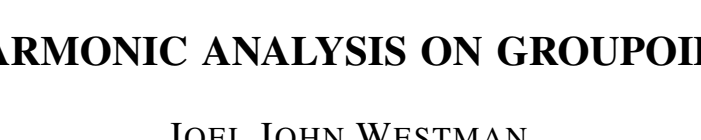




\title{
HARMONIC ANALYSIS ON GROUPOIDS
}

\author{
Joel J. WestMan
}

This paper generalizes harmonic analysis on groups to obtain a theory of harmonic analysis on groupoids. A system of measures is obtained for a locally compact locally trivial groupoid, $Z$, analogous to left Haar measure for a locally compact group. Then a convolution and involution are defined on $C_{c}(Z)=$ the continuous complex valued functions on $Z$ with compact support. Strongly continuous unitary representations of $Z$ on certain fiber bundles, called representation bundles, are lifted to $C_{c}(Z)$, yielding * representations of $C_{c}(Z)$. A norm, \|\|$_{12}$, is defined on $C_{c}(Z)$, and the convolution, involution, and representations all extend to $\mathscr{L}_{12}(Z)=$ the \|\|$_{12}$ completion of $C_{c}(Z)$. The main example given is that of the groupoid $Z=Z(G, H)$ that arises naturally from a Lie group $G$ and a closed subgroup $H$. In this example, the representations of $Z$ are related to induced representations of $G$. Finally, if $Z_{e e}$ (=the group of elements in $Z$ with left unit=right unit $=e$ ) is compact then we canonically represent $\mathscr{L}_{2}(Z)$ as a direct sum of certain simple $H^{*}$-algebras.

We use extensively the notation and results of [8], except that [8] assumes a $C^{r}$ manifold structure on the groupoid $Z$, and we want to consider groupoids with just topological structure. There is no essential difficulty in developing the main results of [8] for locally trivial topological groupoids. In particular, a $C^{r}$ coordinate (resp. $C^{r}$ fiber) bundle in [8] becomes a coordinate (resp. fiber) bundle as defined in [7].

Reviewing $[8, \S 1]$, the algebraic structure of a (transitive) groupoid, $Z$ (over $M$ ), consists of a subset $M$ of $Z$ (called the units of $Z$ ), a projection $l \times r$ of $Z$ onto $M \times M$ sending $\Phi_{q p} \in Z$ into (left unit $\Phi_{q p}$, right unit of $\left.\Phi_{q p}\right)=(q, p)$, and a law of composition defined for pairs $\Phi_{q p}, \Psi_{r s}$ such that $p=r$. For $B \subseteq M \times M, Z_{B}$ is defined as $(l \times r)^{-1}(B)$, and $Z_{q p}=(l \times r)^{-1}(q, p)$. The composition $\Phi_{q p} \cdot \Psi_{p s} \in Z_{q s}$, and $\left(\Phi_{q p} \cdot \Psi_{p s}\right) \cdot \Gamma_{s t}=\Phi_{q p} \cdot\left(\Psi_{p s} \cdot \Gamma_{s t}\right)$. The unit $q \in M$ may be written $1_{q q}$, and $1_{q q} \cdot \Phi_{q p}=\Phi_{q p} \cdot 1_{p p}=\Phi_{q p}$. Also, $\Phi_{q p}$ has an inverse, $\Phi_{q p}{ }^{-1}$, such that $\Phi_{q p}{ }^{-1} \cdot \Phi_{q p}=1_{p p}$ and $\Phi_{q p} \cdot \Phi_{q p}{ }^{-1}=1_{q q}$.

A coordinate groupoid $\left(Z, \Sigma_{e}\right)$ over $M$ consists of the following:

(1.1) An (algebraic, transitive) groupoid $Z$ over $M$ and a Hausdorff topological structure for $M$.

(1.2) A distinguished point $e \in M$ and a Hausdorff topological group structure for the group $Z_{e e}$.

(1.3) A set of functions $\Sigma_{e}=\left\{\alpha: U_{\alpha} \rightarrow Z_{U_{\alpha} \times e}\right\}$ such that $U_{\alpha}$ is 
open in $M$ and $l \cdot \alpha=$ identity map, satisfying

(1.3.1) $\underset{\alpha \in \Sigma_{e}}{\bigcup} U_{\alpha}=M$.

(1.3.2) For $\alpha$ and $\beta \in \Sigma_{e}$, the $\operatorname{map} g_{\alpha \beta}: U_{\alpha} \cap U_{\beta} \rightarrow Z_{e e} ; g_{\alpha \beta}(q)=$ $\alpha(q)^{-1} \circ \beta(q)$, is continuous.

Then the constructions of [8] lead to a topological structure for $Z$, making $Z$ a locally trivial topological groupoid as defined by Ehresmann in [3]. Conversely, any such groupoid arises from a coordinate groupoid.

Finally, we stipulate that the letter " $Z$ " will always represent a locally compact locally trivial groupoid. Note $Z$ is locally compact if and only if both $Z_{e e}$ and $M$ are locally compact.

2. We first consider systems of measures on a groupoid, $Z$ over $M$.

DEFintion 2.1. A (continuous) system of measures on $Z$ is an indexed set $\lambda=\left\{\lambda_{q p}:(q, p) \in M \times M\right\}$, where $\lambda_{q p}$ is a regular Borel measure on $Z_{q p}$. We will write $\lambda_{q p}(f)=\int_{Z} f\left(\Phi_{q p}\right) d \lambda \Phi_{q p}$, where $f$ is an integrable function on $Z_{q p}$, and will require that the function $\lambda(h)$ : $M \times M \rightarrow C ; \lambda(h)(q, p)=\lambda_{q p}\left(\left.h\right|_{z_{q p}}\right)$ be in $C_{c}(M \times M)$ whenever $h \in C_{c}(Z)$.

The concepts of "left and right invariance" are easily applied to systems of measures.

Definition 2.2. A system of measures, $\lambda$, is said to be left invariant if and only if

$$
\begin{gathered}
\int_{Z_{r p}} f\left(\Psi_{q r} \cdot \Phi_{r p}\right) d \lambda \Phi_{r p} \\
=\int_{z_{q p}} f\left(\Gamma_{q p}\right) d \lambda \Gamma_{q p},
\end{gathered}
$$

for all $\Psi_{q r} \in Z$ and $p \in M$ and $f \in C_{c}\left(Z_{q p}\right)$. Similarly, for right invariance the condition is (with $f \in C_{c}\left(Z_{p r}\right)$ ):

$$
\begin{gathered}
\int_{Z p q} f\left(\Phi_{p q} \cdot \Psi_{q r}\right) d \lambda \Phi_{p q} \\
=\int_{z_{p r}} f\left(\Gamma_{p r}\right) d \lambda \Gamma_{p r} .
\end{gathered}
$$

If $Z_{e e}$ is unimodular, it is easy to obtain a left and right invariant system of measures for $Z$ from a Haar measure on $Z_{e e}$ (use (2.6.1) with $\Delta \equiv 1$ ). In the general case, we extend the modular function for $Z_{e e}$ to $Z$, and then obtain a left invariant system of measures for $Z$ (depending on the extension). 
Definition 2.3. A function $\Delta: Z \rightarrow R^{+}$is called a modular function for $Z$ if and only if:

(2.3.1) $\Delta$ is a continuous homomorphism (multiplicative structure for $R^{+}=$real numbers $>0$.)

(2.3.2) $\left.\Delta\right|_{z_{e e}}$ is the modular function for $Z_{e e}$.

THEOERM 2.4. If $M$ is paracompact, then there exists a modular function for $Z$. Given two modular functions, $\Delta$ and $\Delta^{\prime}$, on $Z$, we have $\Delta^{\prime}\left(\Phi_{q p}\right)=h(q, p) \Delta\left(\Phi_{q p}\right), h: M \times M \rightarrow R^{+}$is a continuous homomorphism (with the trivial groupoid structure on $M \times M$, see (3.5b)).

Proof. Let $\Sigma_{e}$ be a set of local sections in $Z_{M x_{e}}$ such that $\left\{U_{\alpha}=\right.$ dom $\alpha: \alpha \in \Sigma_{e}$ \} is a locally finite cover of $M$ (using the paracompactness of $M$ ) and let $\left\{f_{\alpha}\right\}$ be a partition of 1 such that support $\left(f_{\alpha}\right) \cong U_{\alpha} \cdot \Delta_{e e}$ is the modular function for $Z_{e e}$. We define $\Delta=e^{\delta}$, where

$$
\delta\left(\Phi_{q p}\right)=\sum_{f \alpha, f \beta} f_{\alpha}(q) f_{\beta}(p) \log \left(\Delta_{e e}\left(\alpha(q)^{-1} \cdot \Phi_{q p} \cdot \beta(p)\right)\right) .
$$

Then $\Delta$ is a modular function for $Z$. Given a continuous homomorphism $h: M \times M \rightarrow R^{+}$, then $\Delta^{\prime}$ defined by $\Delta^{\prime}\left(\Phi_{q p}\right)=h(q, p) \Delta\left(\Phi_{q p}\right)$ is a modular function for $Z$. Conversely, given two modular functions $\Delta$ and $\Delta^{\prime}$ on $Z$, we find that $h(q, p)=\Delta^{\prime}\left(\Phi_{q p}\right) / \Delta\left(\Phi_{q p}\right)$ is independent of $\Phi_{q p}$ for the given units, and that $h: M \times M \rightarrow R^{+}$is a continuous homomorphism.

THEOREM 2.5. If $\lambda$ is a left (resp. right) invariant system of measures on $Z$, then $\lambda_{q q}$ is a left (resp. right) Haar measure on $Z_{q q}$ for each $q \in M$.

From here on we assume $\lambda_{e e}$ is a fixed left Haar measure on $Z_{e e}$, and will write $\lambda_{e e}(f)=\int_{Z_{e e}} f\left(\Phi_{e e}\right) d \Phi_{e e}$.

THEOREM 2.6. There is a natural one-to-one correspondence between the left invariant systems of measures on $Z$ and the modular functions on $Z$.

Proof. Given a modular function, $\Delta$, on $Z$, we define the system of measures, $\lambda$, by

$$
\begin{aligned}
& \lambda_{q p}(f)=\int_{Z_{q p}} f\left(\Phi_{q p}\right) d \Phi_{q p} \\
& =\int_{Z_{e e}} \Delta\left(\Gamma_{e p}\right) f\left(\Psi_{q e} \Lambda_{e e} \Gamma_{e p}\right) d \Lambda_{e e} .
\end{aligned}
$$


$\lambda_{q p}$ is independent of the choice of $\Psi_{q_{e}}$ and $\Gamma_{e^{p}}$ with the indicated units, and $\lambda$ is left invariant. Conversely if $\lambda$ is a left invariant system of measures the above equation defines $\Delta$ on $Z_{e \times M}$. Then $A$ may be extended to a continuous homomorphism of $Z$ into $R^{+}$, and $\left.\Delta\right|_{Z_{e e}}$ is the modular function of $Z_{e e}$.

THeOREm 2.7. If $Z_{e e}$ is unimodular, then there is a unique left and right system of measures on $Z$ (recall $\lambda_{e e}$ is a fixed left Haar measure).

\section{Proof. Just choose $\Delta \equiv 1$.}

From here on we will assume that a fixed modular function $A$ has been given for $Z$, and the corresponding left invariant system of measures is $\lambda$ as defined in (2.6.1). A fixed regular Borel measure, $\mu$, is specified for $M$, and $\mu(f)$ will be written $\int_{M} f(q) d q$, for any integrable function $f$ on $M$. We require support of $\mu=M$.

3. Definition 3.1. Given $f$ and $g \in C_{c}(Z)$ we define the convolution of $f$ and $g, f^{*} g$, by $f^{*} g\left(\Phi_{q p}\right)=\int_{M} \int_{Z_{q r}} f\left(\Psi_{q r}\right) g\left(\Psi_{q r}{ }^{-1} \cdot \Phi_{q p}\right) d \Psi_{q r} d r$.

THEOREM 3.2. $C_{c}(Z)$ forms an algebra over $C$ with convolution as the law of multiplication, and the usual addition and scalar multiplication.

Proof. The main points to verify are:

(a) $f^{*} g \in C_{c}(Z)$ and

(b) $\left(f^{*} g\right)^{*} h=f^{*}\left(g^{*} h\right)$.

In regard to (a), if support $(f) \subseteq A$ and support $(g) \subseteq B$ then it is easy to show that support $\left(f^{*} g\right) \subseteq A \cdot B . A \cdot B$ is the image of $(A \times B) \cap D \leqq Z \times Z$ under composition, where $D$ is the (closed) subset of $Z \times Z$ where composition is defined. Hence $A \cdot B$ is compact if $A$ and $B$ are compact.

In regard to $(\mathrm{b})$, we compute $\left(f^{*} g\right)^{*} h\left(\Phi_{q p}\right)$

$$
=\int_{M} \int_{Z_{q s}}\left(\int_{M} \int_{Z_{q r}} f\left(\Psi_{q r}\right) g\left(\Psi_{q r}{ }^{-1} \cdot \Gamma_{q s}\right) d \Psi_{q r} d r\right) h\left(\Gamma_{q s}{ }^{-1} \cdot \Phi_{q p}\right) d \Gamma_{q s} d s .
$$

Substitute $\Lambda_{r s}=\Psi_{q r}{ }^{-1} \cdot \Gamma_{q s}$, and interchange the order of integration to obtain

$$
\begin{aligned}
& =\int_{M} \int_{Z_{q r}} f\left(\Psi_{q r}\right)\left(\int_{M} \int_{Z_{r s}} g\left(\Lambda_{r s}\right) h\left(\Lambda_{r s}{ }^{-1} \cdot \Psi_{q r}{ }^{-1} \cdot \Phi_{q p}\right) d \Lambda_{r s} d s\right) d \Psi_{q r} d r \\
& =f^{*}\left(g^{*} h\right)\left(\Phi_{q p}\right) .
\end{aligned}
$$

Next, we define an involution for $C_{c}(Z)$. 
Definition 3.3. Given $f \in C_{c}(Z)$, we define $f^{*}$ by

$$
f^{*}\left(\Phi_{q p}\right)=\bar{f}\left(\Phi_{q p}{ }^{-1}\right) \Delta\left(\Phi_{q p}{ }^{-1}\right)
$$

(where $\bar{f}$ is the complex conjugate of $f$ ).

TheOREm 3.4. The map $f \rightarrow f^{*}: C_{c}(Z) \rightarrow C_{c}(Z)$ is an involution (see [6]).

Proof. The only difficult part is to show $(f * g)^{*}=g^{*} * f^{*}$. We compute

$$
\begin{aligned}
&(f * g)^{*}\left(\Phi_{q p}\right)=\int_{M} \int_{Z_{p r}} \bar{f}\left(\Psi_{p r}\right) \bar{g}\left(\Psi_{p r}{ }^{-1} \cdot \Phi_{q p}{ }^{-1}\right) \Delta\left(\Phi_{q p}{ }^{-1}\right) d \Psi_{p r} d r \\
&\left.=\text { (substituting } \Gamma_{q r}=\Phi_{q p} \cdot \Psi_{p r}\right) \\
& \int_{M} \int_{Z_{q r}} \bar{g}\left(\Gamma_{q r}{ }^{-1}\right) \bar{f}\left(\Phi_{q p}{ }^{-1} \cdot \Gamma_{q r}\right) \Delta\left(\Phi_{q p}{ }^{-1}\right) d \Gamma_{q r} d r=\left(g^{*} * f^{*}\right)\left(\Phi_{q p}\right) .
\end{aligned}
$$

ExAMPLES 3.5. (a) Suppose $M=\{e\}$ and $\mu(1)=1$. Then $Z=Z_{e e}$ is a locally compact group, $f^{*} g$ is the ordinary convolution, and $f \rightarrow f^{*}$ is the usual involution.

(b) Suppose $Z=M^{\prime} \times M^{\prime}$ and $M=$ diagonal of $M^{\prime} \times M^{\prime}$. We define the trivial groupoid structure for $Z$ over $M$ as follows:

$$
l(q, p)=(q, q) \text { and } r(q, p)=(p, p),
$$

composition is given by $(q, p) \cdot(p, r)=(q, r)$, and $(q, q) \rightarrow(q, e)$ gives a global section of $l: Z_{M \times e} \rightarrow M$.

If $M^{\prime}$ is discrete, then $f$ and $g \in C_{c}(Z)$ are matrices indexed by $M^{\prime}$, with a finite number of nonzero entries. If $\mu(\{q\})=1$ for all $q \in M$, and $\lambda_{e e}(1)=1$, then $f^{*} g$ is the matrix composition of $f$ and $g$.

(c) Suppose $G$ is a Lie group and $H$ is a closed subgroup of $G$. We define the homogeneous space groupoid for $G$ and $H, Z(G, H)=$ $Z=\{(q, \Phi, p): \Phi \in G, p$ and $q \in G / H$, and $\Phi p=q\}$. The groupoid structure for $Z$ is given as follows: $M=\{(q, 1, q): q \in G / H\}$ is the set of units, and $q \rightarrow(q, 1, q)$ identifies $M$ with $G / H$ to give $M$ the required topology; $l(q, \Phi, p)=(q, 1, q)$ and $r(q, \Phi, p)=(p, 1, p)$. Composition is defined by $(q, \Phi, p) \cdot(p, \Psi, r)=(q, \Phi \cdot \Psi, r)$; the local sections of $l: Z_{M \times e} \rightarrow M$ come from local sections of $G \rightarrow G / H$ (identifying $G / H$ with $M$ as above, and taking $e=1 H$.): $(e, \Phi, e) \rightarrow \Phi$ is a group isomorphism sending $Z_{e e}$ onto $H$, giving $Z_{e e}$ the required topology.

We note that $Z_{M \times e}$ is essentially the usual principal bundle obtained from $G$ and $H$.

For simplicity we only consider in this paper the case where $\Delta_{I I}$ (the modular function for $H$ ) $=\Delta_{G}$ (the modular function for $G$ ), restricted to $H$. Then, by a theorem in [5, Chapter 10], there is a $G$ 
invariant measure on $M$, which we take for $\mu$. There is a canonical (continuous) homomorphism $\zeta: Z \rightarrow G$, defined by $\zeta(q, \Phi, p)=\Phi$. Note that $\zeta$ maps $Z$ onto $G$, and that $\left.\zeta\right|_{e e}$ is an isomorphism mapping $Z_{e e}$ onto $H$. The above consideration leads to the following:

THEOREM 3.5.1. $\Delta_{G} \cdot \zeta$ is a modular function for $Z$. Unless otherwise mentioned we will always use $\Delta=\Delta_{G} \cdot \zeta$ for $Z(G, H)$.

If $M$ is compact and $\mu(1)=1$, then $\zeta^{*}(f)=f \cdot \zeta \in C_{c}(Z)$ for $f \in C_{c}(Z)$, and we obtain the

THEOREM 3.5.2. $\zeta^{*}: C_{c}(G) \rightarrow C_{c}(Z)$ is a one-to-one* homomorphism (with the usual convolution and involution on $C_{c}(G)$, using a suitable left Haar measure on $G$ ).

Proof. The first point is that $f \rightarrow \int_{M} \int_{Z_{q p}} \zeta^{*}(f)\left(\Phi_{q p}\right) d \Phi_{q p} d p$ (writing $\left.(q, \Phi, p)=\Phi_{q p}\right)$ defines a left invariant measure on $G$ which we take as the desired left Haar measure on $G$. Note, this measure on $G$ is independent of the choice of $q \in M$. Next, we compute

$$
\begin{aligned}
\zeta^{*}(f) * \zeta^{*}(g)\left(\Phi_{q p}\right) & =\int_{Z_{q \times M}} \zeta^{*}(f)\left(\Psi_{q r}\right) \zeta^{*}(g)\left(\Psi_{q r}{ }^{-1} \cdot \Phi_{q p}\right) d \Psi_{q r} d r \\
& =\int_{G} f(\Psi) g\left(\Psi^{-1} \cdot \Phi\right) d \Psi \\
& =(f * g)(\Phi)=\zeta^{*}(f * g)\left(\Phi_{q p}\right), \text { as required. }
\end{aligned}
$$

Finally, for $f \in C_{c}(G)$,

$$
\left(\zeta^{*}(f)\right)^{*}\left(\Phi_{q p}\right)=\left(\zeta^{*}(f)\right)\left(\Phi_{q p}{ }^{-1}\right) \Delta\left(\Phi_{q p^{-1}}\right)=f\left(\Phi^{-1}\right) \Delta_{G}\left(\Phi^{-1}\right)=\zeta^{*}\left(f^{*}\right)\left(\Phi_{q p}\right),
$$

as required.

4. Definition 4.1. A (unitary) representation bundle, $E$, is a fiber bundle with a Hilbert space structure for the fiber $Y$, and group $U(Y)=$ the unitary operators on $Y$ with the strong operator topology.

We note that there is a natural inner product field, $\langle$,$\rangle , on$ $E$. For $q \in M,\langle,\rangle_{q}$ is an inner product on $E_{q}$ defined via any admissable map from the fiber $Y$. Then $\langle,\rangle_{q}$ makes $E_{q}$ a Hilbert space and the unitary maps from $Y$ to $E_{q}$ are the admissable maps from $Y$ to $E_{q}$.

Using the given regular Borel measure, $\mu$, on $M$, we obtain an inner product on $\Gamma_{c}(E)$, the continuous sections in $E$ with compact support. For $\gamma$ and $\delta \in \Gamma_{c}(E)$,

$$
\langle\gamma, \delta\rangle=\int_{M}\left\langle\gamma_{q}, \delta_{q}\right\rangle_{q} d q
$$


The completion of $\Gamma_{c}(E)$ with respect to this inner product is then a Hilbert space, to be called $\Gamma_{2}(E)$.

DEFINITION 4.2. A (strongly continuous) unitary representation $\rho$ of $Z$ on a representation bundle $E$ is a continuous homomorphism $\rho: Z \rightarrow A(E)=$ the (locally trivial) groupoid of admissable maps between the fibers of $E$, such that $\rho$ is the identity map on the units of $Z$ (see [8]).

The main results listed below are obtained essentially as in [8, $\S 4]$.

Theorems 4.3. (a) If $\rho$ is given as in (4.2) then $\left.\rho\right|_{z_{e e}}=\rho_{e} d e-$ fines a unitary representation of $Z_{e e}$ on $E_{e}$.

(b) Given a unitary representation $\rho_{e}$ of $Z_{e e}$ on a Hilbert space $E_{e}$, there is a representation bundle $E^{\prime}$ and representation $\rho^{\prime}$ of $Z$ on $E^{\prime}$ such that $\left.\rho^{\prime}\right|_{z_{e e}} \cong \rho_{e}$ (a unitary equivalence).

(c) Two representations $\rho$ and $\rho^{\prime}$ of $Z$ on $E$ and $E^{\prime}$ respectively are equivalent (as in [8]) if and only if $\left.\left.\rho\right|_{z_{e e}} \cong \rho^{\prime}\right|_{z_{e e}}$.

A groupoid representation, $\rho$, of $Z$ on $E^{\rho}$ defines a representation of the algebra $C_{c}(Z) ; \rho: C_{c}(Z) \rightarrow \mathscr{L}\left(\Gamma_{2}\left(E^{\rho}\right)\right)=$ the bounded linear maps of $\Gamma_{2}\left(E^{\rho}\right)$ into itself.

Definition 4.4. Given $f \in C_{c}(Z)$ and $\gamma \in \Gamma_{c}\left(E^{\rho}\right)$, we define $\rho(f) \gamma$ by $(\rho(f) \gamma)_{q}=\int_{M} \int_{Z_{q p}} f\left(\Phi_{q p}\right) \rho\left(\Phi_{q p}\right) \gamma_{p} d \Phi_{q p} d p$. Alternatively,

$$
\langle\rho(f) \gamma, \delta\rangle=\int_{M} \int_{M} \int_{Z_{q p}} f\left(\Phi_{q p}\right)\left\langle\rho\left(\Phi_{q p}\right) \gamma_{p}, \delta_{q}\right\rangle d \Phi_{q p} d q d p
$$

THEOREM 4.5. $\|\rho(f) \gamma\|_{2} \leqq\|f\|_{12}\|\gamma\|_{2}$, where

$$
\|f\|_{12}^{2}=\int_{M \times M}\left(Z_{q p}\left|f\left(\Phi_{q p}\right)\right| d \Phi_{q p}\right)^{2} d q d p .
$$

Proof. See (5.4). Accordingly $\rho(f)$ extends to a bounded operator on $\Gamma_{2}\left(E^{\rho}\right)$ of norm $\leqq\|f\|_{12}$. $\mathscr{L}\left(\Gamma_{2}\left(E^{\rho}\right)\right)$ has a natural Banach* algebra structure.

THEOREM 4.6. The representation $\rho: C_{c}(Z) \rightarrow \mathscr{L}\left(\Gamma_{2}\left(E^{\rho}\right)\right)$ is a ${ }^{*}$ homomorphism.

Proof. For $f$ and $g \in C_{c}(Z)$, we compute 


$$
\begin{aligned}
& \begin{array}{l}
(\rho(f * g) \gamma)_{q} \\
=\int_{M} \int_{Z_{q p}}\left(\int_{M} \int_{Z_{q r}} f\left(\Psi_{q r}\right) g\left(\Psi_{q r}{ }^{-1} \cdot \Phi_{q p}\right) d \Psi_{q r} d r\right) \rho\left(\Phi_{q p}\right) \gamma_{p} d \Phi_{q p} d p \\
=\text { (substituting } \Gamma_{r p}=\Psi_{q r}{ }^{-1} \cdot \Phi_{q p} \text { and interchanging the } \\
\quad \text { order of integration) }
\end{array} \\
& \quad \int_{M} \int_{Z_{q r}} f\left(\Psi_{q r}\right) \rho\left(\Psi_{q r}\right)\left(\int_{M} \int_{Z_{r p}} g\left(\Gamma_{r p}\right) \rho\left(\Gamma_{r p}\right) \gamma_{p} d \Gamma_{r p} d p\right) d \Psi_{q r} d r \\
& \quad=(\rho(f)(\rho(g) \gamma))_{q} \text { as desired. }
\end{aligned}
$$

Finally, we compute

$$
\begin{aligned}
\left\langle\rho\left(f^{*}\right) \gamma, \delta\right\rangle & =\int_{M \times M} \int_{Z_{q p}} f^{*}\left(\Phi_{q p}\right)\left\langle\rho\left(\Phi_{q p}\right) \gamma_{p}, \delta_{q}\right\rangle_{q} d \Phi_{q p} d p d q \\
& =\int_{M \times M} \int_{Z_{q p}} \bar{f}\left(\Phi_{q p}{ }^{-1}\right) \Delta\left(\Phi_{q p}{ }^{-1}\right)\left\langle\rho\left(\Phi_{q p}{ }^{-1}\right) \delta_{q}, \gamma_{p}\right\rangle_{p} d \Phi_{q p} d p d q \\
\text { (see (5.2.1)) } & =\int_{M \times M} \int_{Z p q} \bar{f}\left(\Psi_{p q}\right)\left\langle\rho\left(\Psi_{p q}\right) \delta_{q}, \gamma_{p}\right\rangle_{p}-{ }_{p} d \Psi_{p q} d p \\
& =\langle\gamma, \rho(f) \delta\rangle, \text { so } \rho\left(f^{*}\right)=\rho(f)^{*} .
\end{aligned}
$$

The following example provides a representation analogous to the left regular representation for groups.

EXAMPLE 4.7. Let $\rho_{e}$ be the strongly continuous unitary representation of $Z_{e e}$ on $\mathscr{L}_{2}\left(Z_{e \times M}\right)$ given by $\left(\rho_{e}\left(\Phi_{e e}\right) f_{e}\right)\left(\Psi_{e p}\right)=f_{e}\left(\Phi_{e e}{ }^{-1} \cdot \Psi_{e p}\right)$. The representation bundle $F$ arising from $\rho_{e}$ and $Z$ may be regarded as $=\cup \underset{q \in M}{\mathscr{L}_{2}}\left(Z_{q \times M}\right)$. The map $f \rightarrow f^{\prime} ; C_{c}(Z) \rightarrow \Gamma_{c}(F)$, defined by $f^{\prime}(q)=\left.f\right|_{q \times M} ^{\substack{q \in M \\ \text { is }}}$ bijective, and $\|f\|_{2}=\left\|f^{\prime}\right\|_{2}$. Accordingly, we can identify $\mathscr{L}_{2}(Z)$ and $\Gamma_{2}(F)$. Given $f$ and $g \in C_{c}(Z)$, then $\rho(f) g^{\prime}=$ $(f * g)^{\prime}$.

5. Definition 5.1. For $f \in C_{c}(Z)$, we define

$$
\|f\|_{12}=\left(\int_{M} \int_{M}\left(\int_{Z_{q p}}\left|f\left(\Phi_{q p}\right)\right| d \Phi_{q p}\right)^{2} d q d p\right)^{\frac{1}{2}} .
$$

\| $\|_{12}$ defines a norm on $C_{c}(Z)$; we complete $C_{c}(Z)$ with respect to \|\|$_{12}$ to form $\mathscr{L}_{12}(Z)$.

To simplify matters, we recall the map: $\lambda: C_{c}(Z) \rightarrow C_{c}(M \times M)$, where $\lambda(f)(q, p)=\int_{z_{q p}} f\left(\Phi_{q p}\right) d \Phi_{q p}$.

Theorem 5.2. $\lambda(f * g)=\lambda(f) * \lambda(g)$ and $\lambda\left(f^{*}\right)=\lambda(f)^{*}$, using the trivial groupoid structure on $M \times M$ over the diagonal of $M \times M$. (on $(M \times M)_{e e}=\{(e, e)\}$ the Haar measure is taken as 1$)$. 
Proof. We write $f_{q p}$ for $\lambda(f)(q, p)$. Then

$$
\begin{aligned}
\lambda(f * g)(q, p) & =\int_{Z_{q p}} \int_{M} \int_{Z_{q r}} f\left(\Psi_{q r}\right) g\left(\Psi_{q r}{ }^{-1} \cdot \Phi_{q p}\right) d \Psi_{q r} d r d \Phi_{q p} \\
& =\int_{M} \int_{Z_{q r}} f\left(\Psi_{q r}\right) g_{r p} d \Psi_{q r} d r \\
& =\int_{M} f_{q r} g_{r p} d r=(\lambda(f) * \lambda(g))\left(\Phi_{q p}\right) .
\end{aligned}
$$

Next, to show $\lambda\left(f^{*}\right)=\lambda(f)^{*}$ we should show

$$
\int_{z_{q p}} f\left(\Phi_{q p}{ }^{-1}\right) \Delta\left(\Phi_{q p}{ }^{-1}\right) d \Phi_{q p}=\int_{z_{q p}} f\left(\Phi_{p q}\right) d \Phi_{p q} .
$$

If $p=q=e$ this is a standard theorem. The extension to the general case is routine, using (2.6.1).

Accordingly, $f \rightarrow \lambda(f)$ defines a $*$ homomorphism. Also, $\|f\|_{12}=$ $\|\lambda(|f|)\|_{2}$, where \|\|$_{2}$ is the $\mathscr{L}_{2}$ norm on $C_{c}(M \times M)$. For $f$ and $g \in C_{c}(M \times M)$ it is easy to show that $\|f * g\|_{2} \leqq\|f\|_{2}\|g\|_{2}$. Finally, we obtain the

Theorem 5.3. Given $f$ and $g \in C_{c}(Z)$ then $\|f * g\|_{12} \leqq\|f\|_{12}\|g\|_{12}$ and $\|f\|=\left\|f^{*}\right\|$.

Proof.

$$
\|\lambda(|f * g|)\|_{2} \leqq\left\|\left.\lambda(|f| *|g|)\right|_{2}=\right\| \lambda(|f|) * \lambda(|g|)\left\|_{2} \leqq\right\| f\left\|_{12}\right\| g \|_{12}
$$

settles the first part, and $\left\|\lambda\left(\left|f^{*}\right|\right)\right\|_{2}=\left\|\lambda(|f|)^{*}\right\|_{2}=\| \lambda\left(|f| \|_{2}\right.$ settles the second part.

Accordingly, the convolution and $\left(^{*}\right)$ involution extend to $\mathscr{L}_{12}(Z)$, making $\mathscr{L}_{12}(Z)$ a Banach algebra with a natural involution. Representations also extend to $\mathscr{L}_{12}(Z)$ as shown below.

Theorem 5.4. For $f \in C_{c}(Z)$ and $\gamma \in \Gamma_{c}(E),\|\rho(f) \gamma\|_{2} \leqq\|f\|_{12}\|\gamma\|_{2}$.

Proof. $\langle\rho(f) \gamma, \rho(f) \gamma\rangle$

$$
\begin{aligned}
& =\int_{M} \int_{M} \int_{M} \int_{Z_{q r}} \int_{Z_{q p}} f\left(\Phi_{q p}\right) \bar{f}\left(\Psi_{q r}\right)\left\langle\rho\left(\Phi_{q p}\right) \gamma_{p}, \rho\left(\Psi_{q r}\right) \gamma_{r}\right\rangle d \Psi_{q p} d \Psi_{q r} d r d p d q \\
& \leqq \int_{M \times M \times M}\left|f_{q p}\right|\left\|\gamma_{p}\right\|\left|f_{q r}\right|\left\|\gamma_{r}\right\| d r d p d q \\
& =\int_{M}\left(\int_{M}\left|f_{q p}\right|\left\|\gamma_{p}\right\| d p\right)\left(\int_{M}\left|f_{q r}\right|\left\|\gamma_{r}\right\| d r\right) d q \\
& \leqq \int_{M}\left(\int_{M}\left|f_{q p}\right|\left\|\gamma_{p}\right\| d p\right)^{2} d q
\end{aligned}
$$




$$
\begin{aligned}
& \leqq \int_{M}\left(\int_{M}\left|f_{q p}\right|^{2} d p \int_{M}\left\|\gamma_{p}\right\|^{2} d p\right) d q \\
& =\|f\|_{12}^{2}\|\gamma\|_{2}^{2} .
\end{aligned}
$$

Accordingly, $\rho$ of $Z$ on $E$ lifts to a *representation of $\mathscr{L}_{12}(Z)$ on $\Gamma_{2}(E)$.

Example 5.5. Suppose $Z=Z(G, H)$ as in (3.5 c), and that $G / H$ is compact and $\mu(1)=1$. Then $\zeta^{*}: C_{c}(G) \rightarrow C_{c}(Z)$ (see (3.5.2) is a norm increasing *homomorphism.

Furthermore, a representation $\rho$ of $Z$ on $E$ defines a representation $\rho^{\prime}$ of $G$ on $\Gamma_{2}(E)$, by $\left(\rho^{\prime}(\Phi) \gamma\right)_{q}=\rho\left(\Phi_{q p}\right) \gamma_{p}$, where $p=\Phi^{-1}(q)$ and $\Phi_{q p}=(q, \Phi, p) . \quad \rho^{\prime}$ is a unitary representation since $\mu$ is invariant under $G$. Then $\rho^{\prime}$ is the induced representation (well known in group theory) from the representation $\rho_{e}$ of $Z_{e e}(\cong H)$ on $E_{e}$. The diagram below, relating $Z$ and $G$, commutes.

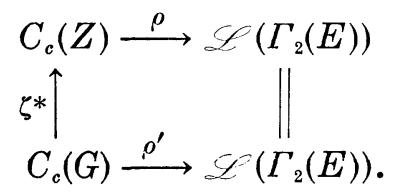

Note that the case $H=G, \mu(1)=\lambda_{e e}(1)=1$, is the same as the Example $3.5 \mathrm{a}$, where $Z=Z_{e e}$.

6. Suppose $Z_{e e}$ is compact, $\Delta \equiv 1$, and $\lambda_{e e}(1)=1$ (the vertically compact case). Then the completion of $C_{c}(Z)$ with respect to the \|\|$_{2}$ norm forms the Hilbert space $\mathscr{L}_{2}(Z)$. We will extend the "orthogonality relations" for compact groups to the above case, and represent $\mathscr{L}_{2}(Z)$ as a direct sum of simple $H^{*}$ algebras.

Definition 6.1. Given $\gamma$ and $\delta \in \Gamma_{c}\left(E^{\rho}\right)$, where $\rho$ is a representation of $Z$ on $E^{\rho}$, we define $T_{\rho \gamma \delta}: Z \rightarrow C$, by

$$
T_{\rho \gamma \delta}\left(\Phi_{q p}\right)=\left\langle\gamma_{q}, \rho\left(\Phi_{q p}\right) \delta_{p}\right\rangle_{q} .
$$

Theorem 6.2. If $\rho_{e}$ and $\rho_{e}^{\prime}$ are irreducible, then

$$
\left\langle T_{\rho \gamma \sigma^{\prime}} T_{\rho^{\prime} \gamma^{\prime} \delta^{\prime}}\right\rangle=\left\{\begin{array}{l}
\frac{\left\langle\gamma, \gamma^{\prime}\right\rangle\left\langle\delta^{\prime}, \delta\right\rangle}{\operatorname{dim} \rho_{e}} \text { if } \rho=\rho^{\prime} \\
0 \text { if } \rho \text { is not equivalent to } \rho^{\prime} .
\end{array}\right.
$$

Proof. Integrating both sides of (6.2.1) over $M \times M$ yields the desired result. 


$$
\begin{aligned}
\int_{z_{q p}} & \left\langle\gamma_{q}, \rho\left(\Phi_{q}\right) \delta_{p}\right\rangle_{q}\left\langle\gamma_{q}^{\prime}, \rho^{\prime}\left(\Phi_{q p}\right) \delta_{p}^{\prime}\right\rangle d \Phi_{q p} \\
= & \left\{\begin{array}{l}
\frac{\left\langle\gamma_{q}, \gamma_{q}^{\prime}\right\rangle\left\langle\delta_{p}^{\prime}, \delta_{p}\right\rangle}{\operatorname{dim} \rho_{e}} \text { if } \rho=\rho^{\prime} \\
0 \text { if } \rho \text { is not equivalent to } \rho^{\prime} .
\end{array}\right.
\end{aligned}
$$

For $q=p=e,(6.2 .1)$ is just the orthogonality relations for compact groups. The proof of (6.2.1) for general $p$ and $q$ is similar to the usual derivation of the orthogonality relations, for example see [1].

Notation. The representations $\rho$ and $\rho^{\prime}$ of $Z$ on $E^{\rho}$ and $E^{\rho^{\prime}}$ respectively will be such that $\rho_{e}$ and $\rho_{e}^{\prime}$ are irreducible. The map $\delta \rightarrow \delta^{*}$ : $\Gamma_{2}(E) \rightarrow \Gamma_{2}(E)^{*}=$ dual of $\Gamma_{2}(E)$, is defined by $\delta^{*}(\gamma)=\langle\gamma, \delta\rangle . \quad \Gamma_{c}(E)^{*}$ is the image of $\Gamma_{c}(E)$ under $\delta \rightarrow \delta^{*}$. The (algebraic) tensor product $\Gamma_{c}\left(E^{\rho}\right) \otimes \Gamma_{c}\left(E^{\rho}\right)^{*}$ many be regarded as a (dense) subalgebra of $C_{\rho}=$ the Schmidt operators on $\Gamma_{2}\left(E^{\rho}\right)$. In particular $\left(\gamma \otimes \delta^{*}\right)(\beta)=\langle\beta, \delta\rangle \gamma$. Conversely, $\alpha$ and $\beta \in C_{\rho}$ can be regarded as elements of the (Hilbert space) tensor product $\Gamma_{2}\left(E^{\rho}\right) \otimes \Gamma_{2}\left(E^{\rho}\right)^{*}$. The inner product on $C_{\rho}$ is defined by $\langle\alpha, \beta\rangle^{\prime}=\langle\alpha, \beta\rangle \operatorname{dim} \rho_{e}$ where $\langle$,$\rangle is the inner product on$ $\Gamma_{2}\left(E^{\rho}\right) \otimes \Gamma_{2}\left(E^{\rho}\right)^{*}$, making $C_{\rho}$ a simple $H^{*}$ algebra.

Theorem 6.4. The canonical map $T_{\rho}: \Gamma_{c}\left(E^{\rho}\right) \otimes \Gamma_{c}\left(E^{\rho}\right) \rightarrow C_{c}(Z)$ defined by $T_{\rho}\left(\gamma \otimes \delta^{*}\right)=T_{\rho \gamma \delta} \operatorname{dim} \rho_{e}$ extends to a ${ }^{*}$ homomorphism and isometry of $C_{\rho}$ into $\mathscr{L}_{2}(Z)$.

Proof. To show $T_{o}$ defines an isometry from $C_{\rho}$ we compute $\left\langle T_{\rho \gamma \delta} \operatorname{dim} \rho_{e}, T_{\rho \gamma^{\prime} \beta} \operatorname{dim} \rho_{e}\right\rangle=\left\langle\gamma \otimes \delta^{*}, \gamma^{\prime} \otimes \beta^{*}\right\rangle \operatorname{dim} \rho_{e}$ (by the orthogonality relations, $)=\left\langle\gamma \otimes \delta^{*}, \gamma^{\prime} \otimes \beta^{*}\right\rangle^{\prime}$ in $C_{\rho}$. In $C_{\rho},\left(\gamma \otimes \delta^{*}\right) \circ\left(\gamma^{\prime} \otimes \beta^{*}\right)(\alpha)$ $=\langle\alpha, \beta\rangle\left\langle\gamma^{\prime}, \delta\right\rangle \gamma$. To show $T_{\rho}$ is a homomorphism we need $T_{\rho \gamma \delta} * T_{\rho \gamma^{\prime} \beta}$ $=\left(\left\langle\gamma^{\prime}, \delta\right\rangle T_{\rho \gamma \beta}\right) / \operatorname{dim} \rho_{e}$. We compute

$$
\begin{aligned}
T_{\rho \gamma \delta} * T_{\rho \gamma^{\prime} \beta}\left(\Phi_{q p}\right) & =\int_{M} \int_{Z_{q r}}\left\langle\gamma_{q}, \rho\left(\Psi_{q r}\right) \delta_{r}\right\rangle\left\langle\gamma_{r}^{\prime}, \rho\left(\Psi_{q r}^{-1} \cdot \Phi_{q p}\right) \beta_{p}\right\rangle d \Psi_{q r} d r \\
& =\int_{M}\left\langle\gamma_{q}, \rho\left(\Phi_{q p}\right) \beta_{p}\right\rangle\left\langle\gamma_{r}^{\prime}, \delta_{r}\right\rangle d r / \operatorname{dim} \rho_{e}=T_{\rho \gamma \beta}\left(\left\langle\gamma^{\prime}, \gamma\right\rangle / \operatorname{dim} \rho_{e}\right)
\end{aligned}
$$

as desired. Finally, it is easy to show that

$$
T_{\rho}\left(\left(\gamma \otimes \delta^{*}\right)^{*}\right)=\left(T_{\rho}\left(\gamma \otimes \delta^{*}\right)\right)^{*} .
$$

THEOREM 6.5. Let $\mathscr{E}$ be a set of irreducible representations of $Z$ containing exactly one member from each equivalence class. Then $\sum_{\rho \in \mathscr{G}} T_{\rho}$ is $a^{*}$ isomorphism and isometry of $\sum_{\rho \in \mathscr{E}} C_{\rho}$ onto $\mathscr{L}_{2}(Z)$. 
Proof. The main point is that the functions $T_{\rho \gamma o}$ for $\rho \in \mathscr{E}, \gamma$ and $\delta \in \Gamma_{c}\left(E^{\rho}\right)$, separate the points of $Z$, and $T_{\rho r \delta}$ is orthogonal to $T_{\rho^{\prime} \gamma^{\prime} \delta^{\prime}}$ if $\rho \neq \rho^{\prime}$ and $\rho$ and $\rho^{\prime} \in \mathscr{E}$.

7. Remarks. 7.0. The algebra $C_{c}(Z)$ forms a quasi-unitary algebra as defined by Dixmier in [2] if we use the inner product

$$
\begin{aligned}
\langle f \cdot g\rangle & =\int_{M} \int_{M} \int_{Z_{q r}} \sqrt{\Delta\left(\Phi_{q p}\right)} f\left(\Psi_{q r}\right) \bar{g}\left(\Psi_{q r}\right) d \Psi_{q r} d q d r, f^{*}=f^{s}, \text { and } \\
f^{j}\left(\Phi_{q p}\right) & =f\left(\Phi_{q p}\right) / V \sqrt{\Delta\left(\Phi_{q p}\right)} .
\end{aligned}
$$

Then $C_{c}(Z)$ is essentially the same as the algebra Dixmier defines on page $310,[2]$ in the special case that $Z$ is the example of (3.5c). Also, in this special case, the representation defined in (4.4) is substantially the same as that defined by Glimm in Theorem 1.5, [4].

The author is indebted to the referee for the above references ([2] and [4]).

\section{REFERENCES}

1. C. Chevalley, Theory of Lie Groups, Princeton University Press, 1946.

2. J. Dixmier, Algèbres quasi-unitaires, Comment. Math. Helv. 26 (1952), 275-321.

3. C. Ehresmann, "Categories topologiques et categories differentiables," Colloque de Geometrie Differentielle Globale, Bruxelles, 1958.

4. J. Glimm, Families of induced representations, Pacific J. Math. 12 (1962), 885-911.

5. S. Helgason, Differential Geometry and Symmetric Spaces, Academic Press, 1962.

6. L. Loomis, Abstract Harmonic Analysis, Van Nostrand, 1953.

7. N. Steenrod, The Topology of Fiber Bundles, Princeton University Press, 1951.

8. J. Westman, Locally trivial $C^{r}$ groupoids and their representations, Pacific J. Math. 20 (1967).

Received July 5, 1967.

UNiversity of California, Irvine 


\section{PACIFIC JOURNAL OF MATHEMATICS}

\section{EDITORS}

\section{H. ROYDEN}

Stanford University

Stanford, California

\section{J. Dugundu}

Department of Mathematics University of Southern California Los Angeles, California 90007

RICHARD ARENS

University of California Los Angeles, California 90024

ASSOCIATE EDITORS
E. F. Beckenbach
B. H. NEUMANN
F. WoLF
K. YosidA

\section{SUPPORTING INSTITUTIONS}

\author{
UNIVERSITY OF BRITISH COLUMBIA \\ CALIFORNIA INSTITUTE OF TECHNOLOGY \\ UNIVERSITY OF CALIFORNIA \\ MONTANA STATE UNIVERSITY \\ UNIVERSITY OF NEVADA \\ NEW MEXICO STATE UNIVERSITY \\ OREGON STATE UNIVERSITY \\ UNIVERSITY OF OREGON \\ OSAKA UNIVERSITY \\ UNIVERSITY OF SOUTHERN CALIFORNIA
}

STANFORD UNIVERSITY

UNIVERSITY OF TOKYO

UNIVERSITY OF UTAH

WASHINGTON STATE UNIVERSITY

UNIVERSITY OF WASHINGTON

AMERICAN MATHEMATICAL SOCIETY
CHEVRON RESEARCH CORPORATION
TRW SYSTEMS

AMERICAN MATHEMATICAL SOCIETY

TRW SYSTEMS

NAVAL WEAPONS CENTER

Mathematical papers intended for publication in the Pacific Journal of Mathematics should be in typed form or offset-reproduced, double spaced with large margins. Underline Greek letters in red, German in green, and script in blue. The first paragraph or two must be capable of being used separately as a synopsis of the entire paper. It should not contain references to the bibliography. Manuscripts, in duplicate if possible, may be sent to any one of the four editors. All other communications to the editors should be addressed to the managing editor, Richard Arens, University of California, Los Angeles, California 90024.

Each author of each article receives 50 reprints free of charge; additional copies may be obtained at cost in multiples of 50 .

The Pacific Journal of Mathematics is published monthly. Effective with Volume 16 the price per volume (3 numbers) is $\$ 8.00$; single issues, $\$ 3.00$. Special price for current issues to individual faculty members of supporting institutions and to individual members of the American Mathematical Society: $\$ 4.00$ per volume; single issues $\$ 1.50$. Back numbers are available.

Subscriptions, orders for back numbers, and changes of address should be sent to Pacific Journal of Mathematics, 103 Highland Boulevard, Berkeley 8, California.

Printed at Kokusai Bunken Insatsusha (International Academic Printing Co., Ltd.), 7-17, Fujimi 2-chome, Chiyoda-ku, Tokyo, Japan.

PUBLISHED BY PACIFIC JOURNAL OF MATHEMATICS, A NON-PROFIT CORPORATION

The Supporting Institutions listed above contribute to the cost of publication of this Journal, but they are not owners of publishers and have no responsibility for its content or policies. 


\section{Pacific Journal of Mathematics \\ Vol. 27, No. 3}

March, 1968

Charles A. Akemann, Invariant subspaces of $C(G) \ldots \ldots \ldots \ldots \ldots \ldots . \ldots 41$

Dan Amir and Zvi Ziegler, Generalized convexity cones and their duals ... . 425

Raymond Balbes, On ( $J, M, \mathrm{~m})$-extensions of order sums of distributive

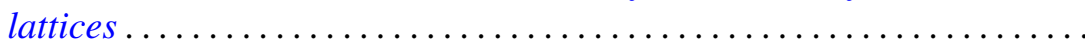

Jan-Erik Björk, Extensions of the maximal ideal space of a function algebra ........................................ 453

Frank Castagna, Sums of automorphisms of a primary abelian group ...... 463

Theodore Seio Chihara, On determinate Hamburger moment problems ..... .

Zeev Ditzian, Convolution transforms whose inversion function has complex

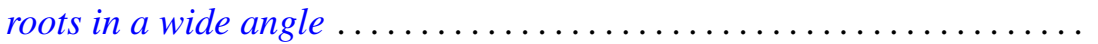

Myron Goldstein, On a paper of Rao .

Velmer B. Headley and Charles Andrew Swanson, Oscillation criteria for

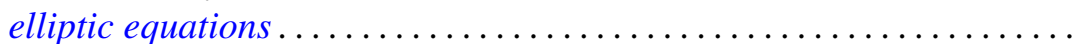

John Willard Heidel, Qualitative behavior of solutions of a third order nonlinear differential equation............................

Alan Carleton Hindmarsh, Pick's conditions and analyticity.............

Bruce Ansgar Jensen and Donald Wright Miller, Commutative semigroups

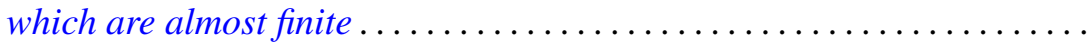

Lynn Clifford Kurtz and Don Harrell Tucker, An extended form of the

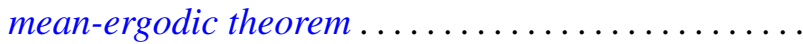

S. P. Lloyd, Feller boundary induced by a transition operator ...

Henry B. Mann, Josephine Mitchell and Lowell Schoenfeld, A new proof of the maximum principle for doubly-harmonic functions ...

Robert Einsohn Mosher, The product formula for the third obstruction ..

Sam Bernard Nadler, Jr., Sequences of contractions and fixed points ...

Eric Albert Nordgren, Invariant subspaces of a direct sum of weighted shifts...

Fred Richman, Thin abelian p-groups ...

Jordan Tobias Rosenbaum, Simultaneous interpolation in $\mathrm{H}_{2}$. II ...

Charles Thomas Scarborough, Minimal Urysohn spaces .

Malcolm Jay Sherman, Disjoint invariant subspaces..... .

Joel John Westman, Harmonic analysis on groupoids....

621

William Jennings Wickless, Quasi-isomorphism and TFM

Minoru Hasegawa, Correction to "On the convergence of resolvents of operators" 